\title{
Reference equation for 6-minute walk in individuals with Parkinson disease
}

\author{
Michael J. Falvo, MS; ${ }^{1}$ Gammon M. Earhart, PT, $\mathbf{P h D}^{1-2^{*}}$ \\ ${ }^{1}$ Program in Physical Therapy and ${ }^{2}$ Department of Anatomy and Neurobiology and Department of Neurology, \\ Washington University School of Medicine, St. Louis, MO
}

\begin{abstract}
Normal values, as well as determining factors, for the 6-minute walk test are available for nondisabled adults, but less is known about individuals with Parkinson disease (PD). This study created a PD-specific reference equation and identified unique factors associated with 6-minute walk distance (6MWD). Eighty individuals (mean $+/-$ standard deviation [SD] age: $66.3+/-9.8 \mathrm{yr}$ ) with mild to moderate PD underwent a neurological examination and completed a small battery of tests to assess their balance confidence, fall risk, mobility, and balance. Their mean +/- SD 6MWD was 394.1 +/- $98.4 \mathrm{~m}$ (95\% confidence interval: 370.0-418.1). Stepwise multiple regression analysis demonstrated that timed up-and-go (TUG) test, one-leg stance (OLS) test, and sex were significant independent contributors to 6MWD and accounted for $56.6 \%$ of the variance. The resulting PD-specific regression equation is $6 \mathrm{MWD}_{\text {pred }}=543.06+(-10.83 \times \mathrm{TUG})+(2.04 \times \mathrm{OLS})+$ $(-44.44 \times$ sex $)$. (For sex, $0=$ female, $1=$ male. $)$ Future interpretation of 6MWD in individuals with PD may be enhanced if expressed as a percentage of the value predicted utilizing this reference equation.
\end{abstract}

Key words: 6-minute walk distance, exercise test, Freezing of Gait questionnaire, functional capacity, one-leg stance test, Parkinson disease, reference equation, timed up-and-go test, UPDRS, walking.

\section{INTRODUCTION}

The 6-minute walk test is widely used in clinical settings to assess functional exercise, often as an outcome measurement for evaluating an intervention [1]. Refer- ence equations for 6-minute walk distance (6MWD) are available [2-3] yet were developed using data from nondisabled older adults. Utilizing these equations for individuals with motor impairment, such as Parkinson disease (PD), may not be appropriate. For example, Garber and Friedman [4] reported 6MWDs in individuals with PD that were 42 percent of that predicted by one such reference equation [2], suggesting that factors unique to PD are not considered in these existing equations. Canning et al. [5], as well as work from our laboratory [6], have identified certain variables that explain large portions of variance in 6MWD and therefore contribute to the observed reduced distances. These variables broadly comprise measures of parkinsonian symptoms and functional mobility (i.e., Berg Balance Score, timed up-and-go [TUG] test), which are distinct from the variables contained in the reference equations for nondisabled adults (e.g., anthropometrics, age, and sex) [2-3].

Abbreviations: $6 \mathrm{MWD}=6$-minute walk distance, $\mathrm{FOG}=$ Freezing of Gait, HY = Hoehn and Yahr, OLS = one-leg stance, PD = Parkinson disease, PIGD = Postural Instability and Gait Disorder, $\mathrm{SD}=$ standard deviation, $\mathrm{TUG}=$ timed up-and-go (test), UPDRS = Unified Parkinson's Disease Rating Scale.

*Address all correspondence to Gammon M. Earhart, PT, PhD; Washington University School of Medicine, Program in Physical Therapy, Campus Box 8502, 4444 Forest Park Ave, St. Louis, MO 63110; 314-286-1425.

Email: earhartg@wustl.edu

DOI:10.1682/JRRD.2009.04.0046 
Given noted features of PD, such as reduced stride length and hypokinesia, 6MWD is reduced in those with PD as compared with nondisabled adults [5,7]. While such a patient-control comparison may be of interest, it may be of additional utility to compare "patients to patients." The latter may be useful in understanding the extent of functional exercise capacity in relation to individuals who share the same neurological disorder. Considering the unique factors associated with 6MWD in PD [5-6], developing predictions specific to individuals with PD would be valuable. As a result, the following comprise the objectives of this study: (1) determine 6MWD in a large sample of individuals with PD, (2) compare measured 6MWD with predicted 6MWD from existing reference equations, and (3) identify factors contributing to 6MWD and develop a PD-specific reference equation. Parts of our results have been reported previously (e.g., demographics, performance scores) [6], but the objective and analysis of this report are distinct.

\section{METHODS}

Eighty individuals with idiopathic PD [8] were recruited from the Washington University Movement Disorders Clinic. Subjects were excluded if they had other neurological deficits or serious medical issues and/ or were unable to independently ambulate. Demographics are provided in the Table. All eligible subjects provided their written informed consent following a health screening and study briefing. Experimental testing was approved by the Human Research Protection Office at the Washington University School of Medicine.

The following data were recorded: (1) anthropometric variables, (2) the Unified Parkinson's Disease Rating Scale (UPDRS) [9], (3) Hoehn and Yahr (HY) scale [10], (4) the Activities-specific Balance Confidence scale [11], (5) the Freezing of Gait (FOG) questionnaire [12], (6) one-leg stance (OLS) test [13], (7) TUG test [14], and (8) the 6-minute walk test [15].

The OLS test assesses balance by having an individual stand on only his or her preferred leg; the single best time out of three attempts was used for analysis. The TUG test is a basic test of functional mobility whereby the individual rises from a chair, walks $3 \mathrm{~m}$, and then returns to sit in the chair. Similarly, we used the single best time out of three attempts. For the 6-minute walk test, subjects were asked to walk as far as they could down a $30.5 \mathrm{~m}$ corridor for 6 minutes. They were advised
Table.

Demographics and performance for 80 participants with Parkinson disease (PD).

\begin{tabular}{lcc}
\hline \multicolumn{1}{c}{ Variable } & Mean \pm SD (Range) & $\boldsymbol{r}$ \\
\hline Sex (No.) & 24 female, 56 male & $-0.33^{* \dagger}$ \\
Age (yr) & $66.3 \pm 9.8(37.0-83.0)$ & $-0.24^{\dagger}$ \\
Height (cm) & $174.2 \pm 10.6(149.9-195.2)$ & 0.20 \\
Weight (kg) & $80.8 \pm 19.4(46.4-156.8)$ & 0.14 \\
PD Duration (yr) & $8.6 \pm 5.1(0.3-20.0)$ & -0.02 \\
HY Stage (1-5) & $2.3 \pm 0.5(1.0-4.0)$ & $-0.40^{* \dagger}$ \\
UPDRS Motor (0-108) & $28.7 \pm 9.4(4.0-49.0)$ & $-0.28^{\dagger}$ \\
PIGD Subscale (0-20) & $2.8 \pm 2.2(0-10.5)$ & $-0.45^{* \dagger}$ \\
ABC Scale (0-100) & $74.3 \pm 20.5(26.2-100.0)$ & 0.02 \\
FOG Questionnaire (0-24) & $7.7 \pm 5.7(0-19.0)$ & $-0.34^{* \dagger}$ \\
OLS Time (s) & $12.3 \pm 15.6(0-60.0)$ & $0.47^{* \dagger}$ \\
TUG (s) & $10.7 \pm 4.7(5.2-36.6)$ & $-0.67^{* \dagger}$ \\
6MWD (m) & $394.1 \pm 98.4(152.7-613.6)$ & - \\
\hline
\end{tabular}

${ }^{*}$ Entered into stepwise regression.

${ }^{\dagger}$ Significantly correlated with 6MWD $(p<0.05)$.

$6 \mathrm{MWD}=6$-minute walk distance, $\mathrm{ABC}=$ Activities-specific Balance Confidence, FOG = Freezing of Gait, HY = Hoehn and Yahr, OLS = one-leg stance, PIGD = Postural Instability and Gait Disorder, $\mathrm{SD}=$ standard deviation, $\mathrm{TUG}=$ timed up-and-go, UPDRS = Unified Parkinson's Disease Rating Scale.

that they could slow down or rest if needed. Spotters were available to supervise the execution of turns at the ends of the corridor. To determine whether subjects also experienced any akinetic blocks or freezing of gait, we also had them complete the FOG questionnaire.

The Postural Instability and Gait Disorder (PIGD) subscale, derived from items 27-30 on the UPDRS motor subsection, was also computed and is thought to represent clinical mobility [16]. The same clinician also rated the clinical disability of each subject according to the HY scale. Scores range from 0 to 5 , with 0 indicating no signs of the disease and 5 being reserved for those who are wheelchair bound or unable to independently move. A score of 2 to 3 generally indicates mild to moderate disease severity and includes bilateral symptoms and postural instability in some cases.

Collectively, these data were hypothesized to capture the variance in 6MWD. One should note that height was not recorded for 38 of our 80 subjects. However, we did have records of subjects' leg length, which has been used previously to estimate height [17]. Using the full data sets (i.e., known heights) from our 42 subjects, we estimated leg length to be 52 percent of total height and then applied this parameter to the 38 subjects whose heights were not directly recorded. (See the "Results" section for additional discussion.) 
All statistical analyses were performed with SPSS v17.0 software (Chicago, Illinois). Pearson correlation coefficients were calculated, and a stepwise multiple regression was used to determine independent variables explaining the variance in 6MWD. An additional forcedentry regression using variables identified in previous literature (e.g., age, height, weight, and sex) [2-3] was also performed for comparison purposes. Variables were allowed to enter the model at the 5 percent level of significance. Data were checked for and met the assumptions of regression (normality, multicollinearity, homoscedasticity, etc.) and are presented as mean \pm standard deviation (SD).

\section{RESULTS}

Considerable variability was noted in the 6MWD (range: 152.7-613.6 m) for individuals with PD (mean age of $66 \mathrm{yr}, \mathrm{HY}$ of 2.3), with an average distance of $394.1 \mathrm{~m}$. Some of this variability may be accounted for through univariate correlation analysis demonstrating sex, age, HY stage, UPDRS, TUG, PIGD, FOG, and OLS to be significantly correlated with 6MWD ( $r=-0.67$ to 0.47, $p<0.05$ ). Coefficients are presented in the Table. As seen in the Table, height is not significantly correlated with 6MWD ( $r=0.20, p=0.22)$ for the entire sample. We ran an additional correlation for those subjects $(n=42)$ for whom height was not estimated and also did not find a significant relationship $(r=0.17, p=0.48)$.

We chose six variables with the largest significant univariate correlations to enter into our stepwise multiple regression (i.e., 16 cases per variable). PIGD, OLS, TUG, FOG, HY, and sex were entered into our stepwise multiple regressions, and TUG, OLS, and sex were identified as significant $(p<0.01)$ independent contributors to 6MWD, explaining approximately 56.6 percent of the variance. The largest portion was explained by TUG (44.7\%), followed by OLS time (7.8\%) and sex (4.0\%). For predicting $6 \mathrm{MWD}\left(6 \mathrm{MWD}_{\text {pred }}\right)$, our regression equation is $6 \mathrm{MWD}_{\text {pred }}=543.06+(-10.83 \times \mathrm{TUG})+(2.04 \times$ OLS $)+(-44.44 \times$ sex $)$. (For sex, $0=$ female, $1=$ male. $)$

Using predictor variables derived from nondisabled adults [2-3], we performed a forced-entry regression analysis and were only able to explain 17.8 percent of the variance in 6MWD in our sample with $\mathrm{PD}$, compared with the 56.6 percent that was explained using a stepwise technique. In addition, we input our data into the existing equations for nondisabled individuals by using their pub- lished coefficients and found these equations to significantly overestimate mean 6MWD by 57.9 percent on average (range: 143.2-254.2 m). To further confirm the appropriateness of our estimated height data, we also separately analyzed those subjects $(n=42)$ with full data sets and found a very similar 58.9 percent overestimation of $6 \mathrm{MWD}$ compared with that observed. With the exception of sex, no variables were common among the previously reported equations and those found in the present study. A comparison of observed distance, predicted distance based on our equation, and predicted distances based on two existing equations developed from nondisabled populations (ages 40-85 yr) is presented in Figure (b).

To illustrate the agreement between our observed scores and predicted scores, a Bland-Altman plot is presented in Figure (a). The mean \pm SD of the differences is $-4.53 \pm 114.25$ and, therefore, mean \pm 2 SD is $(-233.04$, 223.98). From the plot, only $5 / 80$ points (6.2\%) exceed the \pm 2 SD demarcations. Although the difference between mean observed 6MWD (394.1) and predicted 6MWD (389.5) is small, Figure (a) further illustrates the variability in 6MWD in those with PD.

\section{DISCUSSION}

Our main finding demonstrates that for individuals with PD, the variance in 6MWD is not accounted for by anthropometrics, sex, and age, as seen in nondisabled adults. Therefore, utilizing previously published reference equations to predict 6MWD is not appropriate for individuals with PD, a finding that is supported by earlier work [4]. These equations [2-3] were able to explain between 38 and 66 percent of the variance in 6MWD through height, age, weight, and sex in nondisabled adults. Yet when we forced the entry of these variables (e.g., age, height, weight, sex), only 17.8 percent of the variance in 6MWD was explained. However, we report a PD-specific regression equation that identifies increased fall risk, balance, and sex as independent contributors to 6MWD and explains approximately 56.6 percent of the variance. Our findings support those of Canning et al. [5], which underscores the importance of identifying physical impairments associated with functional exercise capacity (i.e., 6MWD) that may be directly targeted for future rehabilitation.

It has been suggested previously that interpretation of 6MWD is enhanced when expressed as a percentage of predicted values as opposed to a single arbitrary distance 
[3]. However, this suggestion assumed that 6MWD reference equations were developed with the population of interest, which was previously not applicable to individuals with PD. Utilizing the reference equation of the present study, future investigations may be able to interpret 6MWD in individuals with PD as a percentage of that predicted. For example, we may consider an abnormal $6 \mathrm{MWD}$ as that which is below the 95 percent confidence interval. In our sample, this would be a distance of less than $370 \mathrm{~m}$, which was found for 29 out of 80 subjects. On average, this equated to a 6MWD that was approximately 40 percent less than that predicted. Such a criterion may be useful for interpreting functional exercise capacity in individuals with PD. Therefore, we believe this PD-specific reference equation may be of utility to clinicians who are trying to gauge where their patients are performing relative to what is expected. In addition, clinicians may be able to provide a realistic therapy goal of attaining a certain percent of predicted 6MWD based on the PD-specific equation, which is more reasonable than a goal derived from data on nondisabled older adults [2-3].

Caution is advised when interpreting these results for several reasons. First, our cross-sectional design may be prone to selection bias and may overestimate 6MWD in those with more advanced PD. Based on our average HY stage, the majority of this sample is mild to moderate in disease progression. Despite this, our subject characteristics exhibited quite a range (see Table) in many of our assessments. Further, this is the largest sample of individuals with PD $(n=80)$ to date reporting 6MWD. A second potential limitation due to our study design is stability or test-retest reliability of the 6-minute walk test. Although reliability is of greater concern in those studies utilizing $6 \mathrm{MWD}$ as an outcome measurement $[7,18]$, the reliability of this test in persons with PD is strong and has been supported in two separate studies (interclass correlation coefficient $=0.95$ [19] and 0.96 [20]). Lastly, the 6minute walk test is most frequently used in pulmonary and cardiac settings. Because a substantial portion of variance (i.e., 43.4\%) remains unexplained, we cannot account for the influence of motivation and preferred walking speed, which may have contributed noise to these data. In addition, more sophisticated cardiopulmonary assessments may provide additional insight for individuals with PD, and this area is worthy of additional research. However, the advantage of the variables included in the reference equation of the present study is that sophisticated technology is not needed to collect such data.
Performance of the 6-minute walk test varies in individuals with $\mathrm{PD}$, as evidenced by the range in 6MWD (i.e., 315-560 m) [4-5,7,17-20] and illustrated in Figure (a). A portion of this variability may be explained through measures of fall risk (e.g., TUG) and balance (e.g., OLS), as well as by sex. This differs from nondisabled adults, for whom anthropometrics and age are of greater importance. As a result, utilizing regression equations intended for
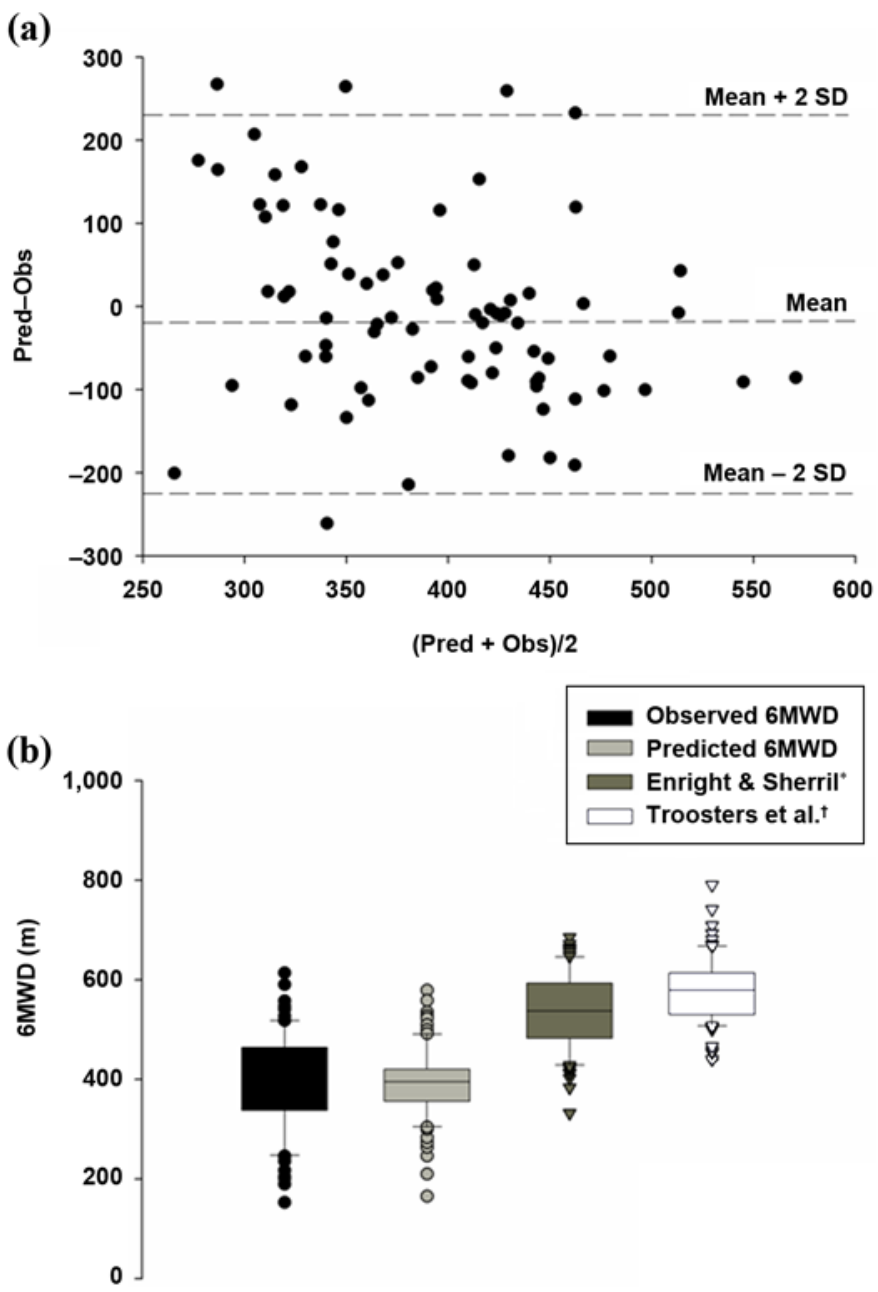

Figure.

(a) Bland-Altman plot illustrating agreement between scores observed (Obs) and scores predicted (Pred) from our equation. SD = standard deviation. (b) Comparison of our sample's observed and predicted 6-minute walk distance (6MWD) with those predicted from equations derived from nondisabled adults. *Enright PL, Sherrill DL. Reference equations for the six-minute walk in nondisabled adults. Am J Resp Crit Care Med. 1998;158(5 Pt 1):1384-87 [PMID: 9817683]. `Troosters T, Gosselink R, Decramer M. Six minute walking distance in nondisabled elderly subjects. Eur Respir J. 1999;14(2):270-74. [PMID: 10515400] DOI:10.1034/j.1399-3003.1999.14b06.x 
nondisabled adults results in inflated 6MWD estimates for individuals with PD (see Figure (b)). Despite our findings, a considerable amount of variability in 6MWD remains to be explained. Canning et al. previously reported that hypokinesia accounted for a large portion of variance in 6MWD in those with PD [5]. These authors quantified hypokinesia as the walking velocity recorded while participants walked as fast as possible over an $8 \mathrm{~m}$ walkway. Unfortunately, we were unable to perform similar measurements to quantify hyopkinetic walking, which may have accounted for a portion of our unexplained variance. However, we suspect that the inclusion of the TUG in the present study provides some utility in describing hypokinesia.

\section{CONCLUSIONS}

The PD-specific reference equation presented herein provides researchers and clinicians the opportunity to interpret their observed 6MWD with more appropriate predicted values. Reference equations for nondisabled adults without PD are inappropriate and do not sufficiently account for the substantial variance in 6MWD for individuals with PD. Alternatively, factors such as fall risk, balance, and sex explain approximately 40 percent more variance than is explained by previously available reference equations. Additional research is necessary to confirm the appropriateness of this equation in other samples and/or identify other factors that help explain a greater amount of variance in 6MWD.

\section{ACKNOWLEDGMENTS}

\section{Author Contributions:}

Study concept and design: M. J. Falvo, G. M. Earhart.

Acquisition of data: G. M. Earhart.

Analysis and interpretation of data: M. J. Falvo.

Drafting of manuscript: M. J. Falvo, G. M. Earhart.

Critical revision of manuscript for important intellectual content:

G. M. Earhart.

Statistical analysis: M. J. Falvo.

Obtained funding: G. M. Earhart.

Administrative, technical, or material support: G. M. Earhart.

Study supervision: G. M. Earhart.

Financial Disclosures: The authors have declared that no competing interests exist.

Funding/Support: This material was based on work supported by the National Institute of Child Health and Human Development (grant T32HD007434). Additional support was provided by grant 1K01HD048437.
Additional Contributions: We would like to thank Madeleine Hackney for subject recruitment. The content is solely the responsibility of the authors and does not necessarily represent the official views of the National Institute of Child Health and Human Development or National Institutes of Health.

Participant Follow-Up: The authors do not plan to inform participants of the publication of this study. However, participants familiar with the laboratory Web site can find the titles (no attachments) of recent publications posted there.

\section{REFERENCES}

1. Du H, Newton PJ, Salamonson Y, Carrieri-Kohlman VL, Davidson PM. A review of the six-minute walk test: Its implication as a self-administered assessment tool. Eur J Cardiovasc Nurs. 2009;8(1):2-8. [PMID: 18694656] DOI:10.1016/j.ejcnurse.2008.07.001

2. Enright PL, Sherrill DL. Reference equations for the sixminute walk in healthy adults. Am J Resp Crit Care Med. 1998;158(5 Pt 1):1384-87. [PMID: 9817683]

3. Troosters T, Gosselink R, Decramer M. Six minute walking distance in healthy elderly subjects. Eur Respir J. 1999; 14(2):270-74. [PMID: 10515400] DOI:10.1034/j.1399-3003.1999.14b06.x

4. Garber CE, Friedman JH. Effects of fatigue on physical activity and function in patients with Parkinson's disease. Neurology. 2003;60(7):1119-24. [PMID: 12682317]

5. Canning CG, Ada L, Johnson JJ, McWhirter S. Walking capacity in mild to moderate Parkinson's disease. Arch Phys Med Rehabil. 2006;87(3):371-75. [PMID: 16500171] DOI:10.1016/j.apmr.2005.11.021

6. Falvo MJ, Earhart GM. Six-minute walk distance in individuals with Parkinson disease: A hierarchical regression model. Arch Phys Med Rehabil. 2009;90(6):1004-8.

[PMID: 19480877]

DOI:10.1016/j.apmr.2008.12.018

7. Köseolu F, Inan L, Ozel S, Deviren SD, Karabiyikolu G, Yorganciolu R, Atasoy T, Oztürk A. The effects of a pulmonary rehabilitation program on pulmonary function tests and exercise tolerance in patients with Parkinson's disease. Funct Neurol. 1997;12(6):319-25. [PMID: 0503194]

8. Calne DB, Snow BJ, Lee C. Criteria for diagnosing Parkinson's disease. Ann Neurol. 1992;32 Suppl:S125-27.

[PMID: 1510370] DOI:10.1002/ana.410320721

9. Movement Disorder Society Task Force on Rating Scales for Parkinson's Disease. The Unified Parkinson's Disease Rating Scale (UPDRS): Status and recommendations. Mov Disord. 2003;18(7):738-50. [PMID: 12815652] DOI:10.1002/mds.10473 
10. Hoehn MM, Yahr MD. Parkinsonism: Onset, progression and mortality. Neurology. 1967;17(5):427-42. [PMID: 6067254]

11. Powell LE, Myers AM. The Activities-specific Balance Confidence (ABC) Scale. J Gerontol A Biol Sci Med Sci. 1995;50A(1):M28-34. [PMID: 7814786]

12. Giladi N, Shabtai H, Simon ES, Biran S, Tal J, Korczyn AD. Construction of freezing of gait questionnaire for patients with Parkinsonism. Parkinsonism Relat Disord. 2000;6(3):165-70. [PMID: 10817956]

DOI:10.1016/S1353-8020(99)00062-0

13. Bohannon RW, Larkin PA, Cook AC, Gear J, Singer J. Decrease in timed balance test scores with aging. Phys Ther. 1984;64(7):1067-70. [PMID: 6739548]

14. Podsiadlo D, Richardson S. The timed "Up \& Go": A test of basic functional mobility for frail elderly persons. J Am Geriatr Soc. 1991;39(2):142-48. [PMID: 1991946$]$

15. Butland RJ, Pang J, Gross ER, Woodcock AA, Geddes DM. Two-, six- and 12-minute walking test in respiratory disease. Br Med J (Clin Res Ed). 1982;284(6329):1607-8. [PMID: 6805625] DOI:10.1136/bmj.284.6329.1607

16. Moses RG. Type 1 diabetes and leg length. Diabetes Res Clin Prac. 2005;70(3):303. [PMID: 15963595] DOI:10.1016/j.diabres.2005.04.006
17. Lozano AM, Lang AE, Galvez-Jimenez N, Miyasaki J, Duff J, Hutchinson WD, Dostrovsky JO. Effect of GPi pallidotomy on motor function in Parkinson's disease. Lancet. 1995;346(8987):1383-87. [PMID: 7475819]

DOI:10.1016/S0140-6736(95)92404-3

Erratum in: Lancet. 1996;348(9034):1108.

18. Dibble LE, Hale TF, Marcus RL, Droge J, Gerber JP, LaStayo PC. High-intensity resistance training amplifies muscle hypertrophy and functional gains in persons with Parkinson's disease. Mov Disord. 2006;21(9):1444-52.

[PMID: 16773643] DOI:10.1002/mds.20997

19. Schenkman M, Cutson TM, Kuchibhatla M, Chandler J, Pieper C. Reliability of impairment and physical performance measures for persons with Parkinson's disease. Phys Ther. 1997;77(1):19-27. [PMID: 8996460]

20. Steffen T, Seney M. Test-retest reliability and minimal detectable change on balance and ambulation tests, the 36item Short-Form Health Survey, and the Unified Parkinson Disease Rating Scale in people with parkinsonism. Phys Ther. 2008;88(6):733-46. [PMID: 18356292]

Submitted for publication April 12, 2009. Accepted in revised form July 2, 2009. 